\title{
Homing Guidance Using Spatially Quantized Signals
}

\author{
Ricardo Bencatel \\ Postdoctoral Researcher \\ University of Michigan \\ Ann Arbor, Michigan, 48109 \\ Email: bencatel@umich.edu \\ Liang Liu \\ Undergraduate Student \\ University of Michigan \\ Ann Arbor, Michigan, 48109 \\ Email: liuliang@umich.edu \\ Anouck R. Girard \\ Associate Professor \\ Department of Aerospace Engineering \\ University of Michigan \\ Ann Arbor, Michigan, 48109 \\ Email: anouck@umich.edu
}

Dave W. Oyler

Ph.D. Candidate

Department of Aerospace Engineering Department of Aerospace Engineering

University of Michigan

Ann Arbor, Michigan, 48109

Email: dwoyler@umich.edu

Danning Sun

Undergraduate Student

Department of Aerospace Engineering Department of Aerospace Engineering

University of Michigan

Ann Arbor, Michigan, 48109

Email: danning@umich.edu

This paper considers homing guidance for a vehicle with a single omnidirectional receiver traveling to a stationary, omnidirectional transmitting beacon by using spatially quantized signal strength measurements. Two homing strategies are presented, and simulations are performed for cases with signal noise and vehicle turn rate limits. The first strategy is the Oyler strategy, which adapts a sliding mode controller and observer from previous work. The second strategy is based on constant heading changes each time a range increment is detected, and this strategy is shown to be suffcient for homing. This study also discusses a signal filter designed to improve the homing controllers' performance. Performance metrics are developed for strategy evaluation and parameter optimization. The performance of each guidance strategy is shown through simulations for a variety of conditions. The Oyler strategy guides the vehicle to the beacon more efficiently than the constant heading change strategy, but it comes with a slight penalty in success rate.

\begin{tabular}{|c|c|}
\hline $\begin{array}{l}\text { Nomenclature } \\
A_{k}\end{array}$ & $k^{\text {th }}$ annulus \\
\hline$d, d^{\min }$ & $\begin{array}{l}\text { Traveled distance prior to conver- } \\
\text { gence and minimum convergence dis- } \\
\text { tance }\end{array}$ \\
\hline$K_{1}, K_{2}$ & Observer gains \\
\hline$M, \hat{M}$ & $\begin{array}{l}\text { Signal filter memory and signal simu- } \\
\text { lator memory }\end{array}$ \\
\hline$N, N_{c}$ & $\begin{array}{l}\text { Total number of Monte Carlo trials } \\
\text { and number of convergent trials }\end{array}$ \\
\hline$n, n_{m}$ & $\begin{array}{l}\text { Number of virtual signal readings and } \\
\text { misclassified readings }\end{array}$ \\
\hline$R_{k}, R_{\max }, \Delta R_{k}$ & $\begin{array}{l}\text { Outer boundary of the } k^{t h} \text { annulus, } \\
\text { boundary of the "no signal region" } \\
\text { and width of the } k^{\text {th }} \text { annulus }\end{array}$ \\
\hline$r_{0}, \hat{r}_{0}$ & $\begin{array}{l}\text { Initial distance to the beacon and ini- } \\
\text { tial estimate of the distance to the bea- } \\
\text { con }\end{array}$ \\
\hline$S$ & $\begin{array}{l}\text { Combined score of success rate and } \\
\text { efficiency }\end{array}$ \\
\hline$t_{\max }$ & Simulation duration \\
\hline$V$ & Forward speed \\
\hline
\end{tabular}




$\begin{array}{ll}w & \text { Weight in the score combination } \\ (x, y) & \text { Vehicle position } \\ \alpha, \beta & \text { Inscribed angles between the diameter } \\ \varepsilon & \text { line and the vehicle path } \\ \eta & \text { Signal error percentage } \\ \theta, \theta_{0}, \hat{\theta}_{0} & \text { Efficiency } \\ & \text { Beacon's Azimuth, initial beacon's } \\ & \text { azimuth and initial estimate beacon's } \\ \lambda, \lambda_{s} & \text { azimuth } \\ \mu_{m}, \mu_{s} & \text { Signal filter ratio and signal simulator } \\ \xi & \text { ratio } \\ \rho & \text { Misclassification rate and success rate } \\ & \text { Convergence angle } \\ \sigma, \sigma_{f}, \sigma_{n}, \hat{\sigma} & \text { Quantized signal strength measure- } \\ & \text { ment reading } \\ & \text { Expected signal strength level, filtered } \\ & \text { signal strength level, received signal } \\ \tau & \text { strength level with noise and virtual } \\ \phi, \phi_{\text {lim }} & \text { signal strength level } \\ \chi & \text { Threshold of the signal simulator } \\ \psi, \psi_{0}, \psi_{c m d}, \Delta \psi & \text { Bank angle and bank limit } \\ \text { Input of the signal simulator } & \text { Heading angle, initial heading an- } \\ \text { gle, heading command, and heading } & \text { change } \\ \omega, \omega_{\text {lim }} & \text { Turn rate and turn rate limit }\end{array}$

\section{INTRODUCTION}

This paper addresses the problem of planar homing guidance, where a vehicle travels toward a stationary beacon using a spatially quantized signal strength measurement. Here, the term spatially quantized signal strength measurement describes a signal strength measurement that takes discrete values as a function of range, i.e., vehicle-beacon relative position. Quantized signals occur in digital communication systems, such as those between Unmanned Air Vehicles (UAVs) and ground stations, where the radio frequency (RF) signal strength is a function of the range between the transmitter and the receiver. Calculating range from these signals requires calibration and exact knowledge of the transmitted signal strength. This work utilizes measurements of only the sign of the range-rate, i.e., the signal strength variation, which can be determined without calibration. Thus, each measurement provides limited information, and measurements can only change at specific locations due to spatial quantization. For simplicity, measurements of this type are referred to as quantized sign of range-rate (QSRR) measurements and the overall measurement system is called a QSRR sensor. The methods proposed allow homing guidance using only standard communication signals as a source of information. The proposed guidance methods are able to home in on a target in GPS denied environments and without resorting to signal direction sensors.

In order to make this guidance strategy applicable to realistic scenarios, which include environmental disturbances, variations in the antenna's RF radiation pattern, etc., this paper considers measurements with noise. Additionally, since this work is motivated in part by its potential use on fixedwing UAVs, which cannot turn instantaneously, it considers turn rate limits.

The key achievement of this paper is to present two guidance strategies that can both guide a vehicle to a beacon using only QSRR signals and turn rate measurements in the presence of measurement noise and vehicle turn rate limits. The first strategy is an implementation of previous work [1,2] that studies signals that are continuous in space, and it accounts for constraints that [1] and [2] do not consider. This strategy is referred to as the Oyler strategy. The second strategy is a novel guidance law based on a constant heading change each time a range increment is detected. This is referred to as the Constant Heading Change strategy (CHC). Sufficient conditions for convergence under this strategy are presented, and it is proven that a heading change of $120^{\circ}$ maximizes the convergence probability. A filter with an optimal filtering ratio is built to reduce the influence of noise. Performance metrics are provided and applied to the two guidance strategies.

\subsection{Motivation}

This paper is motivated by the possibility of extending the capabilities of autonomous vehicles through the utilization of existing components for new purposes, i.e., without the addition of signal direction sensors and in GPS denied environments. Specifically, this work provides methods for homing in spatially quantized signal fields, such as those provided by RF communication systems or simple sonar systems, without requiring any additional sensor or sensor calibration

Consider the following scenario: a civil protection, firefighting, or military unit equipped with UAV assets has field elements and base elements. The base elements have a standard Ground Control Station (GCS), launch and recover the UAVs and control their flight to and from the target sites. The field elements have a Light Ground Station (LGS), e.g., a tablet, through which they receive the UAV's relevant sensor data, e.g., situation evaluation imagery, and issue simple retasking commands. A homing system based on the strength of the communication signals can be used to automatically guide the UAV towards the field elements using only the communication link between the LGS and the UAV. As the homing method does not require explicit localization of the target, i.e., the field element, the LGS does not need to have a localization system, e.g., GNSS. The base team only needs an initial rough estimate to direct the UAV towards the LGS. Moreover, the proposed method does not depend on the content of the communication link, allowing it to work with a high Signal-to-Noise Ratio (SNR) and before a proper data exchange is established.

Several air forces [3-5] and navies [6-8] employ systems that provide quantized signal strength measurements, and are a perfect match for the proposed method. The method could also be used to interact with simple remote sensing systems in agricultural applications, or with sonar systems in underwater vehicles. A different and interesting application is the pointing of a directional antenna, e.g., a 
parabolic or a sector antenna $[9,10]$, towards a broadcasting vehicle with only a roughly known position.

\subsection{Background}

\subsubsection{Literature Review}

Modern homing guidance laws typically make use of the line of sight rate and the time to impact [11,12]. Existing literature also contains many examples of the use of directional techniques to locate omnidirectional transmitting sources. These methods are useful for lower-cost applications or applications with restricted sensor capabilities. For example, Very High Frequency (VHF) radio transmitters are often used to track wildlife [13,14]. Antenna arrays can be used to determine the direction to a transmitting source, and triangulation or direction following can be used for homing. Even with the introduction of GPS trackers, VHF tracking continues to be widely used due to its advantages in cost, transmitter lifetime, and packaging size. Additionally, VHF is often used for the recovery of GPS trackers because it provides long windows for device recovery.

The use of passive acoustic homing was developed for use in naval warfare, especially for torpedoes [15]. It utilizes two hydrophones placed on opposite sides of a vehicle. The body of the vehicle blocks out sound from behind each sensor, and these two directional measurements are crosscorrelated to determine the direction to the acoustic source. This method has also been used to track marine animals [16].

Many approaches to the autonomous underwater vehicle (AUV) homing problem have been implemented. Acoustic systems allow the longest transmission ranges, and they are the most prevalent. Multiple localization methods exist that utilize either multiple beacons or multiple receiving transducers. These include long baseline, short baseline, and ultra-short baseline navigation systems [17]. Optical and electromagnetic techniques have also been developed for the terminal stage of guidance $[18,19]$.

A range-only guidance technique has been proposed as an alternative to bearing-guidance. It offers a simple, lowcost solution that only requires a single beacon and a single sensor [20]. To measure range, the vehicle pings the beacon and measures the time that elapses before the beacon's response is received. A disadvantage of this type of system when compared to systems with multiple transducers is that it is not possible to determine location from a single ping. Additionally, acoustic range measurements can sometimes be inaccurate.

A passive technique for acoustic localization of a beacon with a single, omnidirectional receiver utilizes the closest point of approach (CPA) [21], which occurs when the range-rate changes from negative to positive. At the CPA, the beacon is located along a bearing that is perpendicular to the heading. The beacon's location can be estimated by obtaining bearing lines for at least two different heading angles. Maximum likelihood estimation techniques have been developed for the case of non-ideal measurements to estimate position and gauge the accuracy of the estimation [21]. These techniques are applicable to both stationary beacons and beacons that move linearly.

\subsubsection{Previous Work}

The literature described previously identifies a number of measurements or combinations of measurements that are sufficient to achieve homing guidance, but does not identify necessary measurements. Oyler et al. [1,2] show the sign of the range-rate to be both necessary and sufficient for homing guidance, and present guidance laws that utilize only these measurements. These studies achieve homing guidance without requiring knowledge of the vehicle's location or heading, and they perform well in the presence of measurement noise. Additionally, they do not require the identification of CPAs for multiple heading angles, that require vehicle's location and heading. Thus, other strategies in the literature may achieve better performance, but this performance improvement comes at the cost of more capable sensors. That means that the other strategies proposed in the literature would not be able to provide any meaningful guidance, if provided only the quantized signal strength measurements, i.e., if not equipped with the right sensors or operating in a GPS denied environment.

In [1] and [2], the sign of the range-rate has been proven to be sufficient to guide vehicles with unlimited turn rates to beacons in spatially continuous signal fields. Signal strength quantization and turn rate constraints affect greatly the efficacy of the previously proposed method, in particular under noisy operation conditions. This paper adapts the proposed strategy to handle spatially quantized signal fields, and it addresses vehicles that cannot turn instantaneously. These are major suitability issues when considering the application of the proposed guidance strategy in the real world.

\subsection{Problem Statements}

This study treats the problems defined below.

Given a stationary beacon, a vehicle with unicycle kinematics (Sec. 2.1), and spatially quantized signal strength measurements (Sec. 2.2):

P1 Given additionally that the vehicle might have turn rate limits and that the signal measurement is subject to noise, find a guidance strategy for the vehicle such that its trajectory best satisfies the competing performance metrics (Sec. 4.2).

P2 Given additionally that the vehicle can turn instantaneously and a strategy with a constant heading change $(\Delta \psi)$ each time a range increment is detected (Sec. 3.2), find the limit signal quantization such that the vehicle's convergence towards the beacon is assured.

P3 Given additionally that the vehicle can turn instantaneously and a strategy with a constant heading change $(\Delta \psi)$ each time a range increment is detected (Sec. 3.2), find the $\Delta \psi$ that maximizes the size of the signal quantization regions while ensuring that the vehicle converges towards the beacon. 


\subsection{Original Contributions}

The original contributions of this work are as follows.

C1 New homing strategy consisting of turns with predefined heading angle changes, suited for systems with quantized signal measurements.

C2 Implementation of the Oyler homing strategy in the presence of turn constraints and quantized signal measurements.

C3 Definition of sufficient conditions for homing convergence with the constant heading change strategy, including the angle that optimizes such conditions, and the maximum spatial quantization.

C4 Model for a spatially quantized signal measurement system.

C5 Signal noise filter, to reduce the negative effect of the measurement noise on the homing strategies.

C6 Capabilities and performance comparison among the candidate homing strategies.

Contributions $\mathrm{C} 1$ and $\mathrm{C} 2$ are the guidance strategies to solve $\mathrm{P} 1$. Contribution $\mathrm{C} 3$ builds on $\mathrm{C} 1$ to address problems $\mathrm{P} 2$ and P3. Contribution C4 lays down the signal model used by all Problems. Contribution $\mathrm{C} 5$ improves the performance of the strategies outlined by $\mathrm{C} 1$ and $\mathrm{C} 2$ in the presence of signal noise. Contribution $\mathrm{C} 6$ defines the performance metrics and evaluates the convergence performance to find the guidance strategy that best satisfies the performance metrics, as stated in P1.

\subsection{Paper Structure}

The remainder of this paper is organized as follows: Section 2 provides the vehicle model and the signal measurement system model. Two homing strategies and a signal strength filter are presented in Section 3. The proof for sufficient convergence conditions with the CHC strategy is also included in Section 3. Section 4 describes the Monte Carlo sampling setup and performance metrics that are used to characterize the performance of the controllers. Simulation results and observer gain selection for the Oyler strategy are presented in Section 5. Finally, conclusions and future work are discussed in Section 6.

\section{MODELS}

This section describes the unicycle kinematics model as well as the spatially quantized signal measurement system model.

\subsection{Vehicle Model}

A unicycle model is used to simulate the planar kinematics of the vehicle:

$$
\left\{\begin{aligned}
\dot{x} & =V \cos (\psi), \\
\dot{y} & =V \sin (\psi), \\
\dot{\psi} & =\omega, \text { where } \omega \in\left[-\omega_{\text {lim }}, \omega_{\text {lim }}\right],
\end{aligned}\right.
$$

where the $(x, y)$ and $\psi$ are the vehicle state variables, the position and the heading, respectively. $V$ and $\omega$ are the vehicle controls, the forward speed and the turn rate, respectively. Note that in the motivating scenario in Section 1.1, the UAVs may be capable of sensing and maintaining altitude, and thus two-dimensional space is not an overly restrictive assumption. Two dimensions are also sufficient for many antenna pointing applications. Three-dimensional models may also be considered, and they are discussed in Section 6.2

For a vehicle like a fixed-wing aircraft, the turn rate is constrained by its dynamics. This turn rate limit, $\omega_{\text {lim }}$, is determined by the forward speed and the limit bank angle, $\phi_{\text {lim }}$. For helicopters and quadcopters, since they can make hovering turns, $\omega_{\text {lim }}$ can be $\infty$ :

$$
\omega_{\text {lim }}= \begin{cases}\infty & \text { with unlimited turn rate } \\ \frac{g \tan \phi_{l i m}}{V} & \text { with limited turn rate. }\end{cases}
$$

The coordinate system chosen for this work is the NorthEast-Down (NED) coordinate system.

\subsection{Signal Measurement System Model}

The sensor provides the QSRR signal, $\rho$, which is non-zero at the boundaries of the signal strength annuli, $\left\{R_{k}, k=1, \ldots, N\right\}$, as depicted in Figure 1 . " $A_{k}$ " and " $R_{k}$ " are used to denote the $k^{\text {th }}$ annulus and the $k^{\text {th }}$ boundary radius, respectively. These boundaries are determined by the characteristics of the communication system. The signal measurement therefore is a function of the current vehicle-beacon range and the range-rate. In each annulus, the measurement of the quantized signal strength level, $\sigma$, is used to characterize the signal strength. The signal strength decreases monotonically with the range. There is a range beyond which the signal strength is so weak that it can't be distinguished from the background noise. In these conditions, the communication system indicates no signal ( $\rho=$ null) and the vehicle is considered to be in the "no signal region." $R_{\max }$ is defined as the boundary of the "no signal region." The area within $R_{\max }$ is defined as the "signal region." In the ideal case, the signal strength quantization is:

$$
\sigma= \begin{cases}k & R_{k-1}<r \leq R_{k}, \\ k+1 & r>R_{k}, R_{k}=R_{\text {max }}\end{cases}
$$

The QSRR is defined by:

$$
\rho= \begin{cases}1 & \dot{\sigma}>0 \\ -1 & \dot{\sigma}<0 \\ 0 & \dot{\sigma}=0 \\ \text { null } & r>R_{\max }\end{cases}
$$

Some possible models of boundary radius values are as 


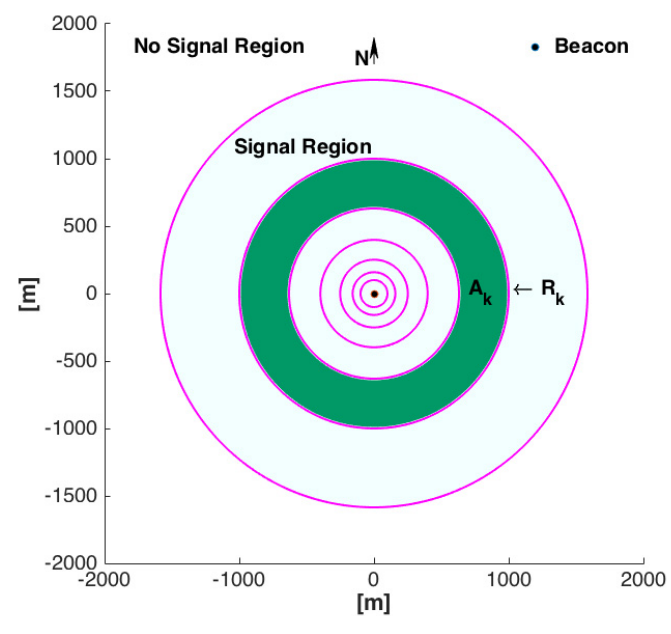

Fig. 1: Graphical representation of signal measurement system model.

follows:

$$
\begin{array}{ll}
R_{k}=a+b k & \forall k, k=1,2,3, \ldots ; a, b \in \mathbb{R}, \\
R_{k}=10^{a+b k} & \forall k, k=1,2,3, \ldots ; a, b \in \mathbb{R},
\end{array}
$$

where $b$ relates to the coarseness of the signal field, which is defined as the annulus width over the outer boundary radius, $\frac{\Delta R}{R}$.

This work focuses on the second model, as it represents a signal strength quantization commonly found in RF communication systems such as 3DR radios [22], those present in UAV autopilots [5] and other autonomous systems [6], namely the Microhard radios [23], which output the RSSI in decibels. Some recreational drones, e.g., Parrot Bebop [24], also provide quantized signal strength measurements for their communication channel with mobile devices, such as iPhones [25]. GSM modems also provide a quantized RSSI measurement that can be converted to decibels [26,27].

The definition of convergence under this signal measurement model is the movement of the vehicle into the innermost annulus, $A_{1}$. Similarly, "single-step convergence" refers to a case where the vehicle moves from its current position into an inner annulus, i.e., when $\sigma^{i-1}=k$ and $\sigma^{i}=$ $k-1$.

Equation (3) holds only for perfect systems. In real systems, signal strength measurements may be noisy, and $\sigma$ might be different from $k$. To simulate realistic conditions, the expected quantized signal strength level, $\sigma$, is corrupted with a signal error probability, $\varepsilon$, which is assumed to be uniform in space. Thus, for a vehicle in annulus $k$, the simulated signal strength level with noise, $\sigma_{n}$, is:

$$
\sigma_{n}= \begin{cases}k & \text { with probability } 1-\varepsilon, \\ k-1 & \text { with probability } \varepsilon / 2 \\ k+1 & \text { with probability } \varepsilon / 2\end{cases}
$$

Note that for $\varepsilon \rightarrow 0, \sigma_{n}$ approaches the ideal case (3).

\section{STRATEGIES}

Two homing strategies are presented; one is an implementation of previous work modified to cope with additional constraints, and the other is new. The main goal of these strategies is to home-in on the beacon while coping with quantized and noisy measurements as well as limited maneuverability, i.e., turn rate. Additionally, a strength filter is presented for use in scenarios with measurement noise.

\subsection{Oyler Strategy}

Oyler et al. [1,2] developed an observer and a heading sliding mode controller that are sufficient to guide vehicles to a beacon, given an unlimited turn rate and spatially continuous signal fields. This section implements this guidance strategy for vehicles with limited turn rates moving in spatially quantized signal fields.

The following are modifications to [1] and [2]:

1. The observer updates the estimate only when the QSRR signal is nonzero.

2. The turn rate command is limited to $\omega_{\text {cmd }} \in$ $\left[-\omega_{\text {lim }}, \omega_{\text {lim }}\right]$.

Note that the estimate is defined relative to the vehicle, and in [1] and [2], updates account for both the new measurement and the motion of the vehicle. Therefore, not updating the estimate when the QSRR signal is zero causes the estimate to move with the vehicle. This overcomes a failure mode caused by signal quantization where the estimate remains fixed in inertial space and the vehicle reaches it. In this case, the vehicle remains in the vicinity of the estimate and may never receive further measurements.

When the QSRR signal becomes null, the vehicle is guided back into the signal region by implementing the "no signal region" strategy described in Section 3.4.

The observer has two observer gains. Among them, the tunable observer gain, $K_{2}$, is an important parameter of the Oyler strategy, because it determines the magnitude of the correction to the beacon estimate's location when the received QSRR reading does not agree with the observer estimate. An interval for the optimal observer gain is determined by Monte Carlo sampling (Sec. 5).

\subsection{Constant Heading Change (CHC) Strategy}

The second strategy, $\mathrm{CHC}$, consists of turning to change the vehicle's heading by a constant, predefined angle each time the vehicle detects a range increment, i.e., when $\sigma^{i}=$ $\sigma^{i-1}+1$. Otherwise, the vehicle maintains its heading. Unlike the Oyler strategy, $\mathrm{CHC}$ requires no estimate of the beacon's location. The only parameter of this guidance law is the value by which the heading changes, $\Delta \psi$.

CHC is defined by Algorithm 1, where $\psi_{c m d}^{i}$ is the heading command at time index $i$.

The CHC strategy is proven to be a sufficient condition for convergence as long as all annuli satisfy a maximumwidth constraint, (Sec. 3.2.1), where annulus width is $\Delta R_{k}=$ $R_{k}-R_{k-1}$. 


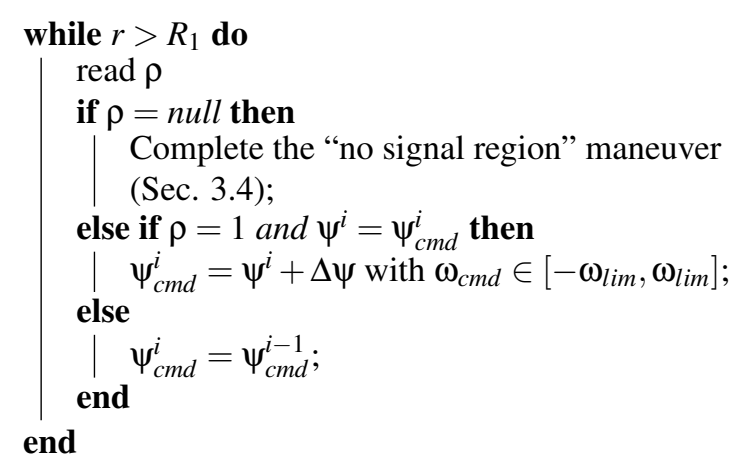

Algorithm 1: CHC strategy logic.

\subsubsection{Sufficient Conditions for Convergence}

This section considers the relationship between $\mathrm{CHC}$ turn angle and minimum annulus width, and it provides a sufficient condition for convergence to the beacon.

Theorem 1. Given a vehicle following a CHC strategy with $\omega_{\text {lim }}=\infty, \varepsilon=0$, and a turn angle of $\Delta \psi$, if the minimum annulus width satisfies

$$
\left.\frac{\Delta R}{R}\right|_{k}< \begin{cases}1-\cos \frac{\Delta \psi}{2} & \Delta \psi \leq \frac{2 \pi}{3} \\ 1+\cos \Delta \psi & \text { otherwise }\end{cases}
$$

then convergence is guaranteed $\left(R=R_{k}\right.$, and $\Delta R=R_{k}-$ $\left.R_{k-1}\right)$.

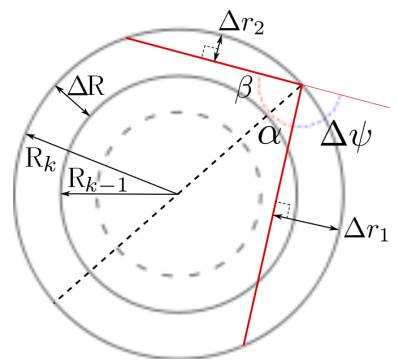

(a) Random scenario.

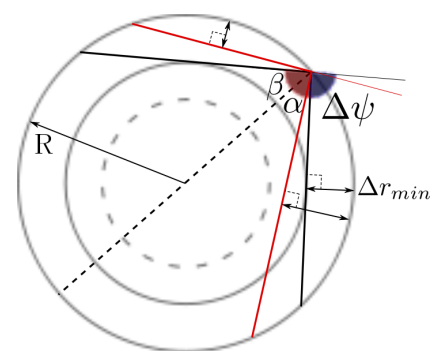

(b) Worst case scenario: bisection.

Fig. 2: Constant Heading Change (CHC) strategy worst case scenario for constant turn angles $\Delta \psi \leq 90^{\circ}$.

Proof. Consider a vehicle following a CHC strategy that enters $A_{k}$ from the outer boundary, as shown in Figure 2a. The vehicle begins on the upper-left side of the figure and travels down and to the right until reaching the edge of annulus $k$, where it turns by an angle of $\Delta \psi$. Let the inscribed angles between the two segments of the vehicle's path and the circle's diameter be $\alpha$ and $\beta$.

First, consider the case where $\beta+\Delta \psi>\pi / 2$ such that the vehicle remains in $A_{k}$ after the turn. Let the maximum distances between the outer boundary of $A_{k}$ and the two segments of the vehicle's path be $\Delta r_{1}$ and $\Delta r_{2}$.

Note the following:

$$
\begin{aligned}
\Delta r_{1} & =R(1-\sin \alpha), \\
\Delta r_{2} & =R(1-\sin \beta), \\
\pi & =\Delta \psi+\alpha+\beta .
\end{aligned}
$$

Single-step convergence occurs if

$$
\max \left(\Delta r_{1}, \Delta r_{2}\right)>\Delta R
$$

1. For a fixed $\Delta \psi \in(0, \pi / 2), \max \left(\Delta r_{1}, \Delta r_{2}\right)$ is minimized when

$$
\alpha=\beta=\frac{\pi-\Delta \psi}{2}
$$

Substituting (10) into (8) gives

$$
\Delta r_{1}=\Delta r_{2}=R\left(1-\cos \frac{\Delta \psi}{2}\right)
$$

This worst-case scenario occurs when both segments of the path are tangent to the inner annulus boundary (Fig. 2b). Taking (9) with (11) implies that single-step convergence is guaranteed as long as

$$
\Delta R<R\left(1-\cos \frac{\Delta \psi}{2}\right)
$$

2. If $\pi / 2<\Delta \psi<\pi$, it is possible for both segments of the vehicle's path to lie on the same side of the line passing through the beacon's position and the location of the turn. As shown in Figure 3, in this scenario $\max \left(\Delta r_{1}, \Delta r_{2}\right)$ is minimized when the paths are such that the length of one of the segments goes to zero. In the limit, the vehicle turns twice at the same location. In this case, $\Delta r_{2} \rightarrow 0$, and the effective maximum distance, $\Delta r_{2}^{\prime}$, becomes the maximum distance between the annulus boundary and the third segment of the vehicle's path. The resulting behavior is then equivalent to the previous scenario with an effective turn angle of $\Delta \psi^{\prime}=2 \pi-2 \Delta \psi$. Then,

$$
\begin{aligned}
\Delta r_{1}=\Delta r_{2}^{\prime} & =R\left(1-\cos \frac{\Delta \psi^{\prime}}{2}\right), \\
& =R(1+\cos \Delta \psi) .
\end{aligned}
$$




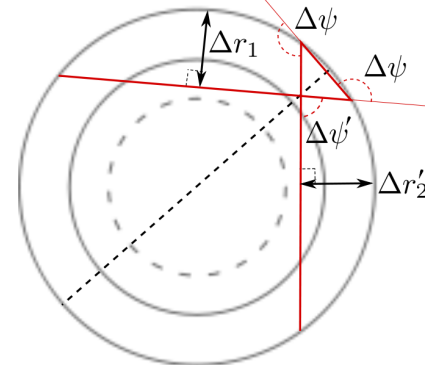

Fig. 3: Constant Heading Change (CHC) strategy worst case scenario for constant turn angles $\Delta \psi \geq 90^{\circ}$.

3. The minimum annulus width for which single-step convergence is guaranteed is given by:

$$
\Delta R_{\min }=R \min \left(1-\cos \frac{\Delta \psi}{2}, 1+\cos \Delta \psi\right)
$$

The two values are equal for

$$
\cos \Delta \psi=-\cos \frac{\Delta \psi}{2} \Leftrightarrow \Delta \psi=\frac{2 \pi}{3} .
$$

Furthermore, this is the only point where the two expressions are equal, because for $\Delta \psi \in[0, \pi)$, the first expression is strictly increasing, and the second expression is strictly decreasing. Thus, the minimum annulus width that guarantees convergence is

$$
\Delta R_{\text {min }}=R \begin{cases}1-\cos \frac{\Delta \psi}{2} & \Delta \psi \leq \frac{2 \pi}{3} \\ 1+\cos \Delta \psi & \text { otherwise }\end{cases}
$$

\subsubsection{Optimal Turn Angle}

Equation (16) gives the minimum allowable annulus width for a given turn angle. Theorem 2 builds on this result to provide the turn angle that maximizes the allowable coarseness in signal quantization.

Theorem 2. Given a vehicle following a CHC strategy with $\omega_{\text {lim }}=\infty$ and $\varepsilon=0$, the annulus width that assures convergence is maximized for $\Delta \psi=120^{\circ}$ and is $\Delta r=r / 2$.

Proof. Equation (16) consists of two parts; the first is strictly increasing, and the second is strictly decreasing. Thus, the maximum is achieved at the switching point between the two expressions:

$$
\underset{\Delta \psi}{\operatorname{argmax}} \frac{\Delta R}{R}=\frac{2 \pi}{3} .
$$

\subsection{Signal Strength Filter}

A signal strength filter is developed to reduce the influence of noise. Each new measurement of signal strength with noise, $\sigma_{n}^{i}$, is combined with the filter memory, $M^{i-1}$, with weights determined by a filtering ratio, $\lambda$, to generate a new filter memory, $M^{i}$. $M^{i}$ is rounded to the nearest integer to determine the estimate of the signal strength level, $\sigma_{f}^{i}=\left[M^{i}\right]$ :

$$
M^{i}=\lambda \cdot M^{i-1}+(1-\lambda) \cdot \sigma_{n}^{i}
$$

where $i$ is the time index of the filter, and $\lambda \in[0,1]$. QSRR is determined by comparing $\sigma_{f}^{i}$ to $\sigma_{f}^{i-1}$.

The performance of the filter depends on $\lambda$, and it is evaluated through simulation by generating a random virtual signal strength level, $\tilde{\sigma}^{i}$, and comparing $\tilde{\sigma}^{i}$ to the filtered version, $\sigma_{f}^{i}$. The misclassification rate, $\mu_{m}$, which is the rate of discrepancy between $\tilde{\sigma}^{i}$ and $\sigma_{f}^{i}$, is used to evaluate the filter performance. The optimal $\lambda$ corresponds to the lowest misclassification rate.

For a sample of $n$ virtual signal readings with $n_{m}$ misclassified readings, the misclassification rate is given by:

$$
\mu_{m}=\frac{n_{m}}{n}
$$

The signal simulator is used to generate a virtual signal strength level. It takes an input, $\chi$, which is randomly selected between two adjacent signal strength levels, $\sigma_{p}$ and $\sigma_{p}+1$, and combines it with the signal simulator memory, $\tilde{M}$, with weights determined by the signal simulator ratio, $\lambda_{g}$, to get a new memory value:

$$
\tilde{M}^{i}=\lambda_{g} \cdot \tilde{M}^{i-1}+\left(1-\lambda_{g}\right) \cdot \chi .
$$

The new virtual signal strength level, $\tilde{\sigma}^{i}$, is then given by:

$$
\tilde{\sigma}^{i}= \begin{cases}\sigma_{p} & \tilde{M}^{i} \leq\left(\sigma_{p}+0.5-\tau\right) \text { and } \tilde{\sigma}^{i-1}=\sigma_{p}+1, \\ \sigma_{p}+1 & \tilde{M}^{i} \geq\left(\sigma_{p}+0.5+\tau\right) \text { and } \tilde{\sigma}^{i-1}=\sigma_{p}, \\ \tilde{\sigma}^{i-1} & \text { otherwise }\end{cases}
$$

where $\tau$ is a threshold that influences the time that the virtual signal stays at each signal strength level.

To get a reasonable switching frequency for $\tilde{\sigma}^{i}, \lambda_{g}$ is set to be 0.95 , and $\tau$ is chosen as 0.1 so that on the average 250 readings are taken between signal changes. The number of readings is based on the characteristics of the model used in this work.

Figure 4 shows the relationship between $\lambda$ and $\mu_{m}$ for filtering ratios between 0 and 1 in increments of 0.01 . Each curve shows the average of 2000 trials for each $\lambda$, and the sample size of the virtual signal in each trial is 15000 . $\varepsilon$ is set from $5 \%$ to $50 \%$ to test performance under different 
noise levels, and the optimal filtering ratio is in the range $[0.8,0.9]$ where misclassification rate is low and also stable with respect to variations in $\varepsilon$.

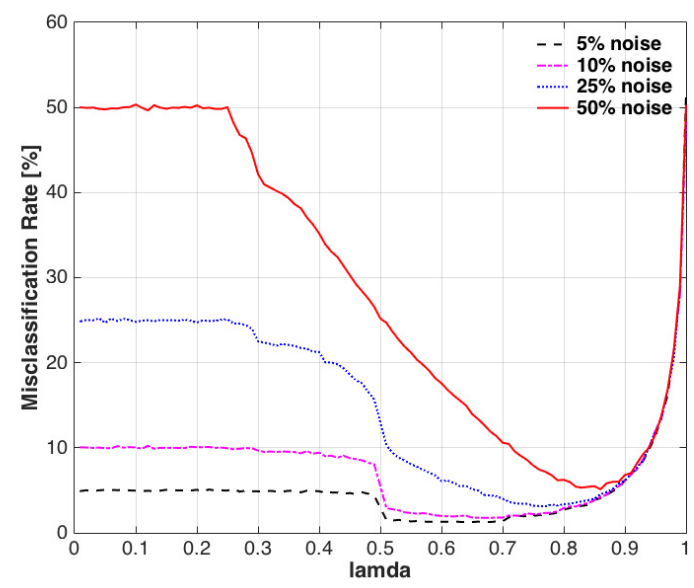

Fig. 4: Misclassification rate.

\section{4 "No Signal Region" Strategy}

This fallback strategy is triggered when the vehicle detects that it has entered the "no signal region" and aims at guiding the vehicle back to the "signal region." For a fair comparison, the same "no signal region" strategy is used with the Oyler and the CHC strategies. No strategy was found that fully assured reentry into the "signal region", in particular for vehicles relying on dead-reckoning. The presented strategy is based on the CHC strategy, due to its convergence characteristics with an optimal heading change of $120^{\circ}$ (Sec. 3.2.2).

The "no signal region" strategy implements the following maneuvering logic:

1. Change the vehicle heading by an angle of $240^{\circ}$ at a rate of $\omega_{\text {lim }}^{\prime}$;

2. Move straight for a distance of $\frac{\sqrt{3} V}{\omega_{\text {lim }}^{\prime}}$.

While performing the maneuver, the system disregards all QSRR measurements. Note that if $\omega_{\text {lim }}=\infty$, a finite $\omega_{\text {lim }}^{\prime}$ needs to be chosen to maintain the desired behavior, else $\omega_{\text {lim }}^{\prime} \leq \omega_{\text {lim }}$.

The first maneuver changes the heading by a complement angle of $120^{\circ}$, i.e., the $\Delta \psi^{\prime}=2 \pi-2 \Delta \psi$ described in the second point of the proof of Theorem 1 . The second maneuver drives the vehicle to produce a trajectory that overlaps itself at point 4 (Fig. 5), which is expected to be inside the "signal region". Point 1 is where the vehicle crosses from the "signal region" to the "no signal region." Point 2 is where it first detects $\rho=$ null and starts turning. Point 3 is where it stops turning.

Note that the use of the signal filter will create a measurement lag that might lead to points 1 and 2 not being coincident. Note also that the distance between points 2 and

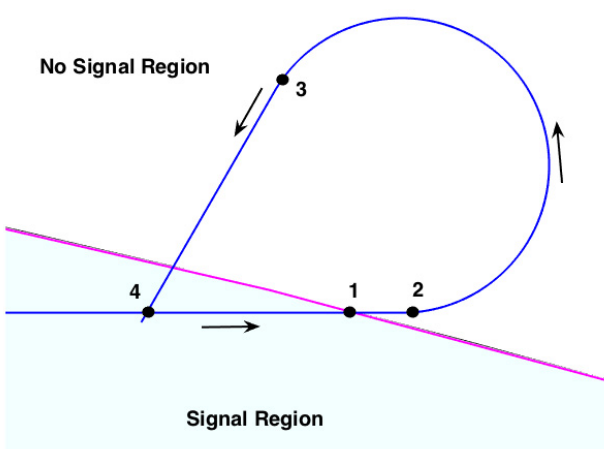

Fig. 5: Graphical representation of the "no signal region" strategy.

4, and points 3 and 4 is $\frac{\sqrt{3} V}{\omega_{\text {lim }}^{\prime}}$ (Fig. 5), which is governed by the choice of $\omega_{\text {lim }}^{\prime}$. As such, the following conditions need to be met to yield a high probability of point 4 being inside the "signal region":

$$
\begin{aligned}
& d_{p_{1}, p_{2}}<\frac{\sqrt{3} V}{\omega_{\text {lim }}^{\prime}}, \\
& \frac{\sqrt{3} V}{\omega_{\text {lim }}^{\prime}}<<R_{\text {max }},
\end{aligned}
$$

where $d_{p_{1}, p_{2}}$ is the distance between points $p_{1}$ and $p_{2}$.

\section{PERFORMANCE METRICS}

A Monte Carlo sampling method is utilized to evaluate the two homing strategies. This section describes the initialization procedure as well as the performance metrics used to evaluate the homing performance.

\subsection{Monte Carlo Sampling Setup}

A Monte Carlo method relies on random sampling. It is used to obtain numerical evaluations of the homing strategies' success rates and efficiencies. Numerical evaluation was chosen because it is difficult to derive theoretical values of these metrics. In this Monte Carlo sampling, the origin of the coordinate system is on the vehicle, and the directions of the axes are aligned with the Earth frame. Due to the symmetry of the annuli, the direction of zero azimuth is defined in the direction of the beacon, $\theta_{0}=0$. Initial conditions then consist of the vehicle heading, the azimuth of the beacon estimate, the distance to the beacon and the distance to the beacon estimate. Initial values are distributed as follows:

1. The vehicle heading, $\psi_{0}$, is uniformly distributed in $\left(-180^{\circ}, 180^{\circ}\right]$, and is a multiple of $0.1^{\circ}$;

2. The azimuth of the beacon estimate, $\hat{\theta}_{0}$, is uniformly distributed in $\left(-180^{\circ}, 180^{\circ}\right]$, and is a multiple of $0.1^{\circ}$;

3. For the distance to the beacon, $r_{0}$, the initial annulus $A_{k}$ is chosen uniformly across all annuli with $R_{1}<R_{k} \leq$ $R_{\text {max }}$. Then $r_{0}$ is randomly selected with uniform distribution in the range $\left(R_{k-1}, R_{k}\right)$ and is a multiple of $0.1 \mathrm{~m}$; 
4. The distance to the beacon estimate, $\hat{r_{0}}$, is uniformly distributed in $\left(10 \mathrm{~m}, R_{\max }\right]$, and is a multiple of $0.1 \mathrm{~m}$.

Note that the minimum distance to the beacon estimate is constrained to $10 \mathrm{~m}$ to avoid numerical errors.

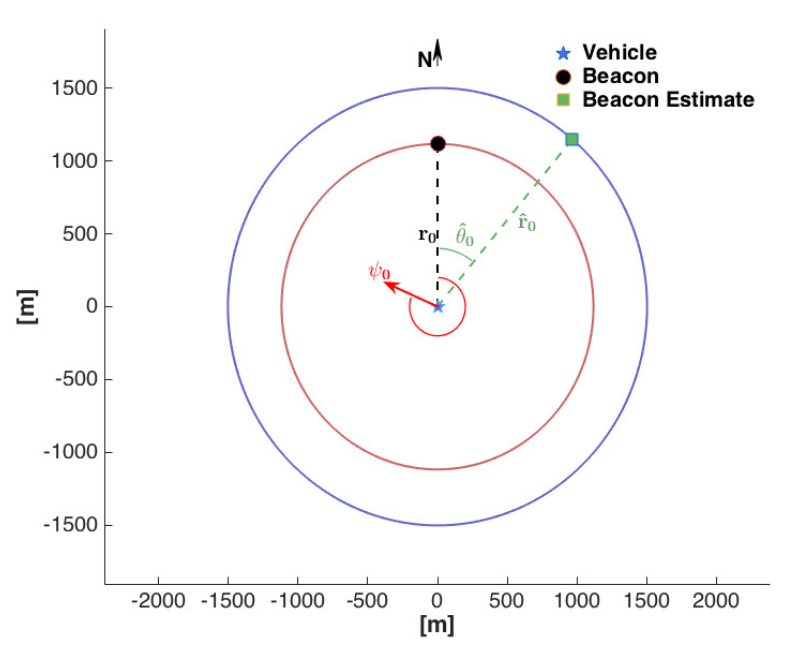

Fig. 6: Monte Carlo sampling initialization.

Trials are terminated when either the vehicle converges or a simulation duration, $t_{\max }$, is reached.

\subsection{Metrics}

Two metrics, success rate, $\mu_{s}$, and efficiency, $\eta$, are used to evaluate the two homing strategies presented in Section 3. They are also used to determine the optimal gain for the Oyler strategy. These metrics are defined as follows:

1. Success Rate: $\mu_{s}$ is the ratio of the number of convergent trials to the number of total trials:

$$
\mu_{S}=\frac{N_{c}}{N}
$$

A guidance law is said to achieve active convergence if its success rate is greater than $50 \%$. This value is chosen because it is the upper limit for the probability of singlestep convergence for arbitrary motion (Fig. 7). For a vehicle in $A_{k}$, if the convergence angle, $\xi$, is the angle between the two tangents from the vehicle's location to the inner boundary of $A_{k}$, as shown in Figure 7 , then $\xi<\pi$ and a random choice of heading has at most a $50 \%$ probability of single-step convergence. Furthermore, unless the vehicle's initial position is in $A_{2}$, convergence requires multiple occurrences of single-step convergence, and therefore the probability of convergence is much lower than $50 \%$. Thus, a success rate greater than $50 \%$ reflects active convergence.

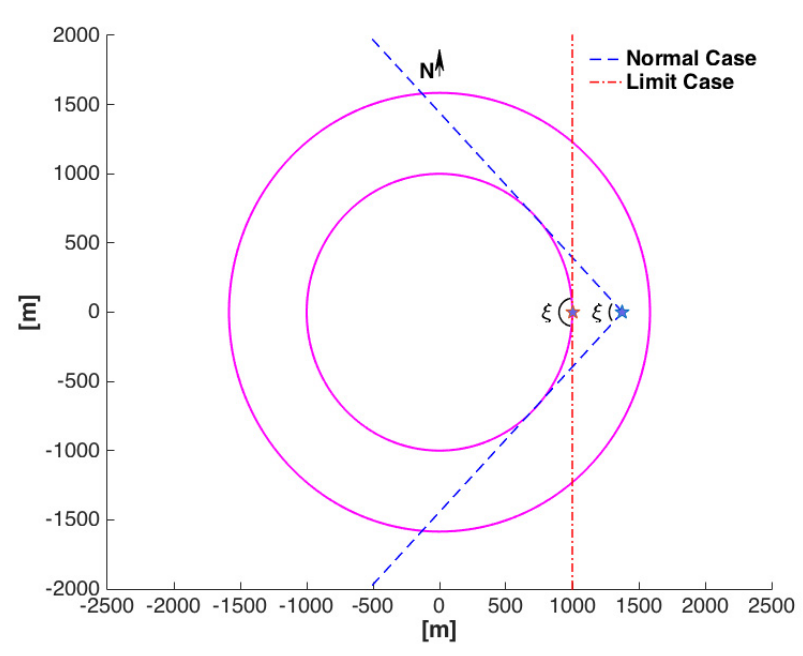

Fig. 7: Quantized signal strength model probability of single-step convergence less than or equal to $50 \%$.

2. Efficiency: The efficiency of trial $j$ is defined as the ratio of $d_{j}^{\text {min }}$ to $d_{j}$, i.e., the minimum distance to $A_{1}$ over the trajectory length prior to reaching $A_{1}$. If the vehicle fails to converge within $t_{\max }$ in a trial, then $d_{j}$ is treated as infinite, so the efficiency of that trial is 0 . The efficiency of a guidance law is given by the mean value of efficiency over all trials:

$$
\eta=\frac{1}{N} \sum_{j=1}^{N} \frac{d_{j}^{\text {min }}}{d_{j}}
$$

\section{RESULTS}

This section shows how well the two proposed homing strategies perform. It begins by presenting the parameter values and conditions selected for the simulations. It then compares the performance of the homing strategies in terms of their success rates and efficiencies. The performance of the Oyler strategy is analyzed as a function of its observer gain, and an observer gain that yields a good performance is selected. Finally, it is shown that the two homing guidance laws are applicable to signal fields with larger sizes and more annuli than the simulated example, with no degradation of guidance performance.

\subsection{Simulation Setup}

Based on flight data obtained with Piccolo autopilots [5], the sensor model used is that defined by (5), with $a=1.8, b=$ 0.2 , and $k=1,2, \ldots, 7$. Simulation results are provided for the following four cases:

1. Base case: $\varepsilon=0$ and $\omega_{\text {lim }}=\infty$;

2. Turn limit case: $\varepsilon=0$ and $\omega_{\text {lim }}=30^{\circ} / \mathrm{s}$;

3. Noise case: $\varepsilon=5 \%$ and $\omega_{\text {lim }}=\infty$;

4. Noise and turn limit case: $\varepsilon=5 \%$ and $\omega_{\text {lim }}=30^{\circ} / \mathrm{s}$. 
For cases with noise, we let $\lambda=0.90$ based on considerations discussed in Section 3.2.1 and from experimental results. Although the simulation shows results only for $\varepsilon=5 \%$, increments in $\varepsilon$ have only minimal influence on the guidance performance (Fig. 4). This is mainly due to the use of the noise filter. In the Monte Carlo Sampling, the number of total trials is 12000 , and $t_{\max }$ is 1500 seconds. The vehicle parameters are chosen to be $V$ is $10 \mathrm{~m} / \mathrm{s}$, and $\omega_{\text {lim }}=30^{\circ} / \mathrm{s}$, which corresponds to a bank limit of $\phi_{\text {lim }} \approx 30^{\circ}$.

\subsection{Simulation Results}

Since success rate is in a binomial distribution with probability close to $100 \%$, the Wilson score interval [28] is used to calculate the confidence interval. In all four simulated cases, the CHC strategy achieves a $99 \%$ confidence level for success rates in the interval $\mu_{S} \in[99.94 \%, 100 \%]$. This results from a $100 \%$ success rate for 12000 trials. This is as expected from the theoretical analysis (Sec. 3.2), because $\Delta R / R=0.369$, which is lower than the critical value of 0.5 for $\Delta \psi=120^{\circ}$.

For the base case, with an observer gain $K_{2}=2^{11}$, the Oyler strategy achieves a 99\% confidence level for success rates in the interval $\mu_{S} \in[97.59 \%, 98.26 \%]$. With larger tested gains both limits of the confidence interval increase. Noise and turn rate limits negatively affect the performance of the Oyler strategy. In the worst case scenario, with an observer gain of $K_{2}=2^{13}$, a $99 \%$ confidence level can be achieved for success rates in the interval $\mu_{S} \in[87.57 \%, 89.08 \%]$. As stated previously, cases are considered unsuccessful if the vehicle is not able to converge onto the inner-most annulus when the maximum time $\left(t_{\max }\right)$ is reached.

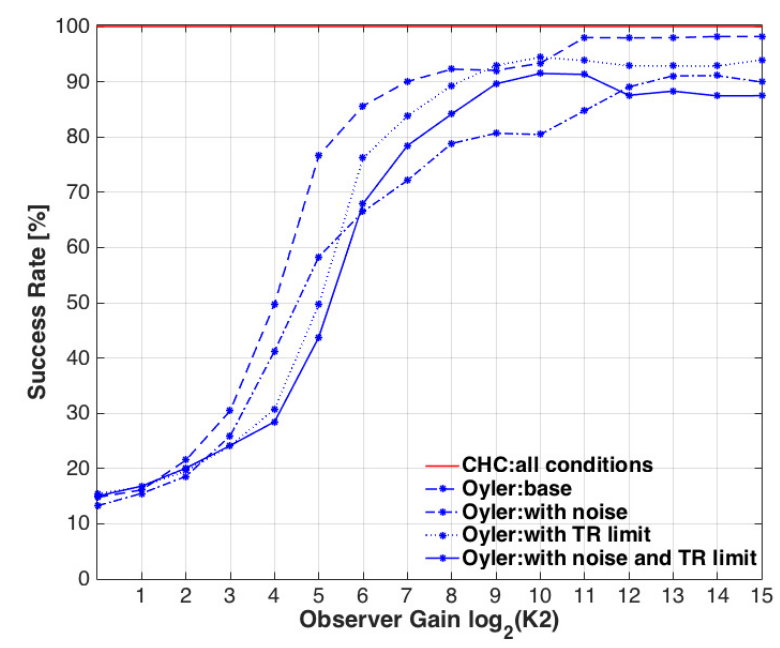

Fig. 8: Success Rate.

Although the success rate with the Oyler strategy is always slightly lower than with the CHC, its efficiency is greater if proper gains are used. The Oyler strategy with an observer gain higher than $2^{7}$ yields greater efficiency than the CHC strategy. The highest efficiencies are obtained with observer gains in the interval $K_{2} \in\left[2^{12}, 2^{13}\right]$. In any of the four cases, the maximum efficiency is at least $65 \%$ greater than that of the CHC strategy.

Also, as Figure 9 shows, noise and turn rate limits have only minimal effects on efficiency for the $\mathrm{CHC}$ strategy. The efficiencies for all four simulated cases are within $1 \%$ of each other. The results for the Oyler strategy also show only a $5 \%$ drop in efficiency when noise and turn rate limits are introduced.

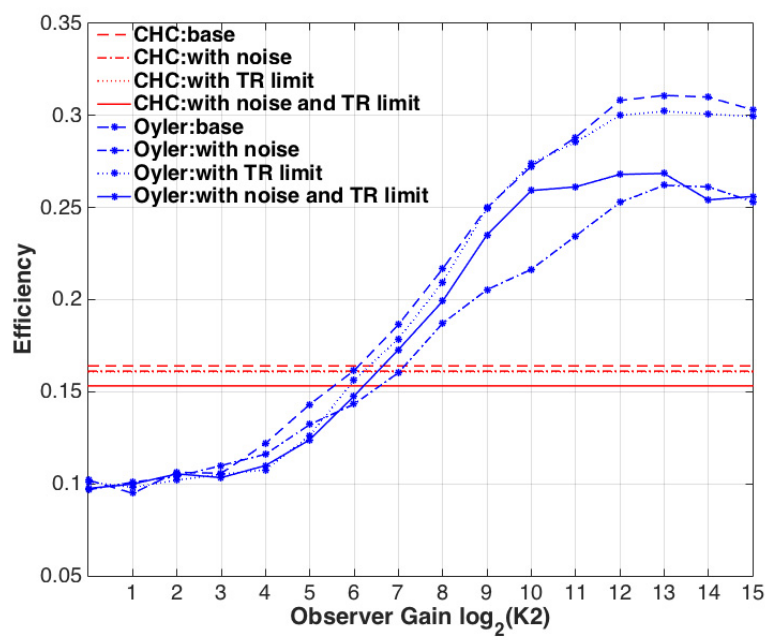

Fig. 9: Efficiency.

Since the optimal observer gain for the two metrics is not necessarily the same, Equation (25) combines both metrics in a single expression that can be used to find a single optimal gain. The two metrics can be prioritized by selecting a weight, $w \in[0,1]$, to account for different applications:

$$
S=(1-w) \cdot \mu_{S}+w \cdot \eta \text {. }
$$

Combinations for different weights are shown in Figure 10, where each curve represents a different value for $w$. Since efficiency usually has a lower numerical value than success rate, giving equal priority to both metrics requires the weights to be chosen unevenly. Nevertheless, there is a strong agreement in that the optimal observer gain should be in the interval $K_{2} \in\left[2^{12}, 2^{15}\right]$.

Figure 11 illustrates two example homing trajectories, one with the Oyler strategy and the other with the CHC strategy. Both trajectories have the same initial conditions and are subject to noise and turn rate limitation. The arrow starting at the diamond represents the initial vehicle heading, while the diamond, star, and asterisks represent the initial vehicle position, the initial beacon estimate, and the final vehicle positions, respectively. 


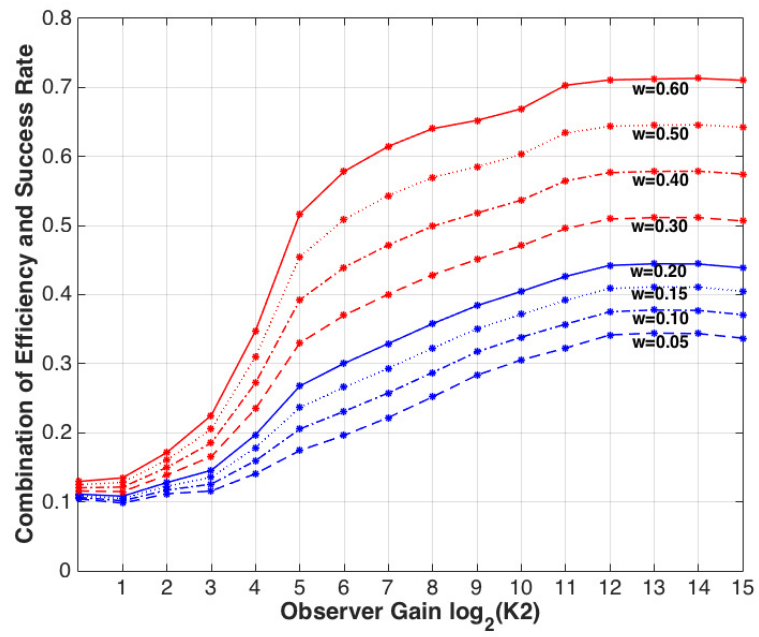

Fig. 10: Combination of efficiency and success rate for base case.

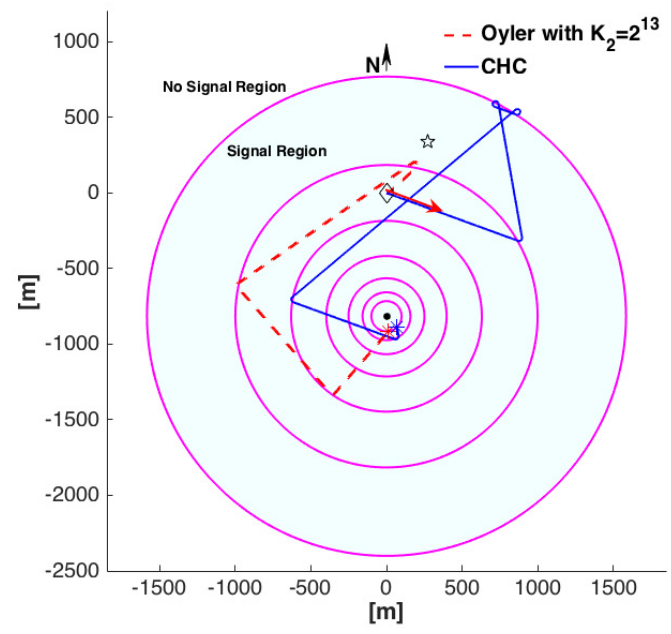

Fig. 11: Convergent trajectories.

These results show that, if the priority is to converge as fast as possible, the Oyler strategy should be used. If the priority is to avoid any unsuccessful convergence attempts, e.g., to avoid loosing the object of interest, then the CHC strategy should be used. The main reason why the CHC strategy is so much less efficient on average, when compared with the Oyler strategy, is that, depending on the preset turning direction $\left(120^{\circ}\right.$ to the right or left) and the initial target bearing relative to the vehicle, the homing trajectory might reach all the way to the outer-most annulus before starting to converge. Some logic can be implemented to check if after a turn the vehicle is converging or diverging, and reverse the course if necessary, which would greatly improve the CHC strategy efficiency, but that is beyond the scope of this paper. The variable turn angle generated by the Oyler strategy might contribute to its efficiency results, but it is also the cause of its slightly lower success rate when compared with the CHC strategy.

\subsection{Extension of the Simulation Results}

In order to evaluate the guidance performance in more general conditions, such as signal fields with different coarseness or a different size $\left(R_{\max }\right)$ from the simulations described in Section 5.2, this section analyzes guidance performance in an individual annulus by using measures of singlestep success rate and efficiency. These single-step metrics are analogous to the two full convergence metrics. In the definitions, the inner boundary of the initial annulus $\left(R_{k-1}\right)$ replaces $R_{1}$, and the trial terminates when the vehicle reaches this inner boundary, i.e., when single-step convergence is achieved. To characterize the system through these metrics, the simulation iterates through different values of coarseness and inner boundary radius. For each Monte Carlo trial, the signal field is defined from the selected annulus width and inner boundary radius, and no constraint is placed on maximum annulus radius. $t_{\max }$ is chosen in proportion to the annulus width.

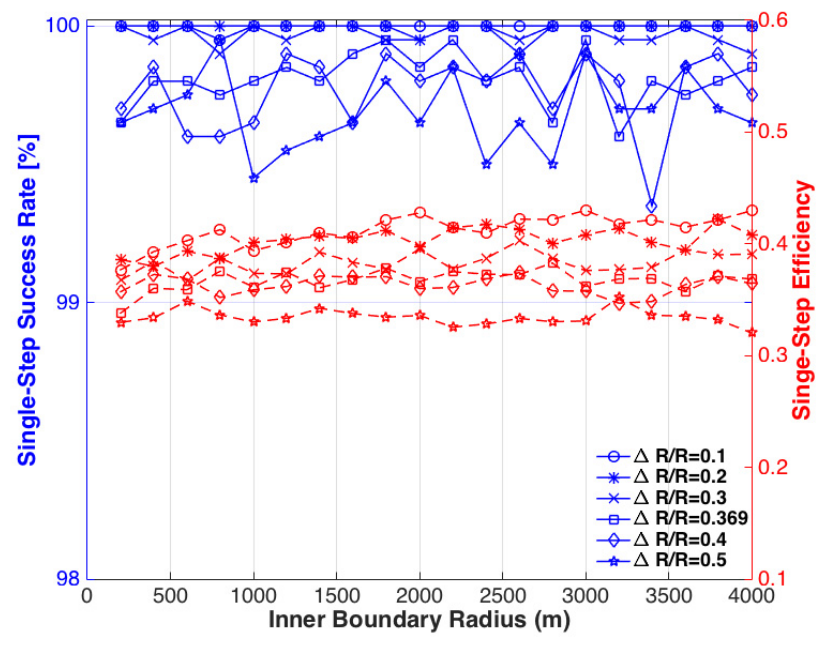

Fig. 12: Single-step success rate and efficiency for the Oyler strategy with $K_{2}=2^{13}$ in base case.

Simulation results show that single-step success rates are almost the same in different annuli. Figures 12 and 13 show results for the Oyler and CHC strategies, respectively, and both figures show single-step efficiencies with dashed curves and single-step success rates with solid curves. As the figures show, the choice of model parameters $a, b$, and $R_{\max }$, has little effect on both homing strategies' success rates, provided that the coarseness is less than or equal to the critical value, 0.5. For comparison, the cases corresponding to the simulation in Section 5.2 are also plotted and have $\Delta R / R=0.369$. Additionally, the single-step efficiency increases for both strategies as coarseness decreases, and it shows no change with inner boundary radius. This is as expected, because as the signal coarseness decreases, the ve- 


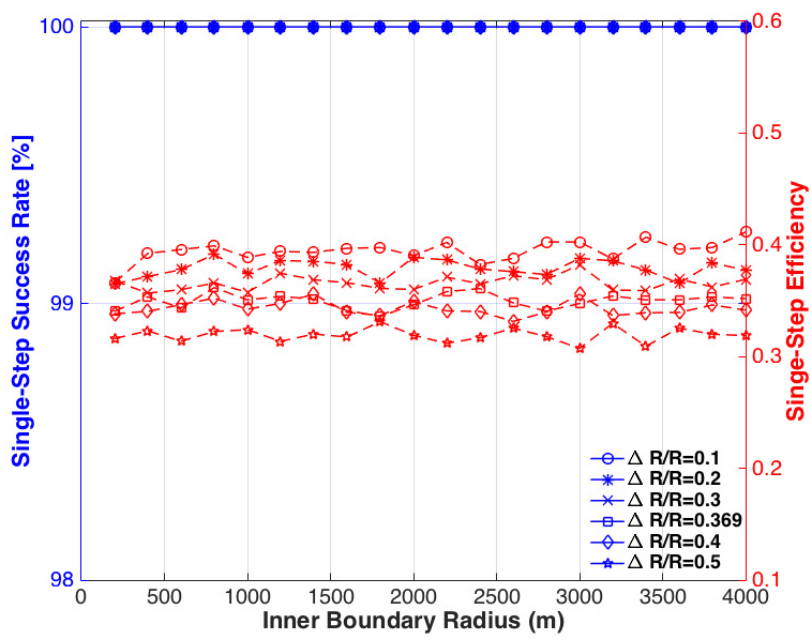

Fig. 13: Single-step success rate and efficiency for the CHC strategy in base case.

hicle obtains information about the beacon's location more frequently, which leads to more efficient trajectories.

This shows that the results obtained in Section 5.2 are relevant for a wide range of communication model parameter values.

\section{CONCLUSIONS \& FUTURE WORK}

\subsection{Conclusions}

This work shows that even though QSRR sensors provide very limited information, these measurements are sufficient for vehicles to successfully home onto beacons. This paper presents two strategies, Oyler and $\mathrm{CHC}$, and both are able to guide vehicles to actively converge to beacons in spatially quantized signal strength measurement fields. Furthermore, both strategies perform well in the presence of noise and turn rate limits.

The Oyler strategy, with proper observer gains, shows good performance, achieving efficiencies $65 \%$ greater than $\mathrm{CHC}$ with only a slight penalty in success rates.

If the success rate is the most important factor, then $\mathrm{CHC}$ is the most appropriate strategy. The $\mathrm{CHC}$ strategy achieves a $100 \%$ success rate and maximizes the allowable signal coarseness, permitting a spatial quantization coarseness up to 0.5 . In fact the most important point made with the $\mathrm{CHC}$ method is that there are sufficient conditions for homing with quantized signal strength measurements. As shown in Section $3.2 .1,120^{\circ}$ is the turn angle that allows for the largest spatial quantization coarseness value, $\Delta R_{\min } / R=0.5$. In these conditions there is a fair chance that the Oyler strategy will converge, but convergence is only assured with the CHC strategy. Finally, noise and turn constraints are shown to have minimal effects on performance.

\subsection{Future Work}

Ongoing work has implemented the proposed strategies in a higher fidelity simulator as a preparatory step for UAV field tests, and this has shown promising preliminary results. Future work will implement the strategies with UAVs using their communication radios.

The signal strength simulation model realism can be improved for RF communications by taking into account the geometry of the radiation pattern of different antennas. This would allow the study of the effect of the aircraft attitude and 3-dimensional position relative to the beacon antenna.

Future work will also consider discreteness in time to make the strategies applicable to realistic digital systems.

It would also be interesting to study how the Oyler and $\mathrm{CHC}$ methods could be combined to produce a homing method with $100 \%$ success rate and high convergence efficiency. The $\mathrm{CHC}$ strategy efficiency can also be improved by itself by adjusting the heading change angle online according to the state of the convergence. Additionally, the strategy adopted in the situations where the vehicle reaches the "no-signal region" can be studied and optimized to minimize the probability of not being able to return to the "signal region", even in GPS denied conditions. A similar strategy can be studied to react to the cases when the aircraft turns in the wrong direction upon reaching $R_{k}$, i.e., when it turns towards the outer annulus instead of the inner annulus.

Given the diversity of homing guidance algorithms for low cost systems based on signal strength or direction, a literature review would be a useful tool in the selection a suitable homing algorithm for a set of available sensors and depending on the expected GNSS signal availability.

\section{ACKNOWLEDGEMENTS}

The authors would like to recognize the significant intellectual contributions of Pierre Kabamba (deceased) to the research leading to this article. The research was supported in part by the United States Air Force grant FA 8650-07-23744 .

\section{References}

[1] D. W. Oyler, P. T. Kabamba, and A. R. Girard, "A homing guidance law for binary range-rate measurements," in Decision and Control (CDC), 2013 IEEE 52nd Annual Conference on, Dec 2013, pp. 1295-1300.

[2] _ , "Binary range-rate measurements and homing guidance," Journal of Dynamic Systems, Measurement, and Control, vol. 137, no. 4, April 2015. [Online]. Available: http://dx.doi.org/10.1115/1.4028527

[3] C. Marshall, M. Mears, D. Kingston, and S. Rasmussen, "2010 ICE-T cooperative control flight testing," in Infotech@Aerospace 2011. AIAA, Mar. 2011. [Online]. Available: http://dx.doi.org/10.2514/6.20111486

[4] T. Oliveira, G. Cruz, E. Marques, and P. Encarnação, "A test bed for rapid fight testing of uav control algorithms," in Research, Development and Education on 
Unmanned Aerial Systems Conference, Seville, Spain, Nov.-Dec. 2011.

[5] B. Vaglienti, R. Hoag, M. Niculescu, J. Becker, and D. Miley, Piccolo User's Guide, v2.1.2 ed., Cloud Cap Technology, Hood River, Oregon, October 122011. [Online]. Available: www.cloudcaptech.com

[6] M. L. Incze, "Lightweight autonomous underwater vehicles (auvs) performing coastal survey operations in rep 10a," Ocean Dynamics, vol. 61, no. 11, pp. 1955-1965, Nov. 2011. [Online]. Available: http://link.springer.com/article/10.1007\%2Fs10236011-0446-z

[7] M. Faria, J. Pinto, F. Py, J. Fortuna, H. Dias, R. Martins, F. Leira, Johansen, J. Sousa, and K. Rajan, "Coordinating uavs and auvs for oceanographic field experiments: Challenges and lessons learned," in Robotics and Automation (ICRA), 2014 IEEE International Conference on, May 2014, pp. 6606-6611. [Online]. Available: http://dx.doi.org/10.1109/ICRA.2014.6907834

[8] I. I. Kaminer, O. A. Yakimenko, V. N. Dobrokhodov, and K. D. Jones, "Rapid flight test prototyping system and the fleet of uavs and mavs at the naval postgraduate school," in AIAA 3rd "Unmanned Unlimited" Technical Conference, Workshop and Exhibit. AIAA, Sep. 2004. [Online]. Available: http://dx.doi.org/10.2514/6.2004-6491

[9] RocketDish, J1071715 ed., Ubiquiti Networks, Inc., July 2015. [Online]. Available: https://dl.ubnt.com/datasheets/rocketdish/rd_ds_web.pdf

[10] airMAX Sector, JL051613 ed., Ubiquiti Networks, Inc., May 2013. [Online]. Available: http://dl.ubnt.com/datasheets/airmaxsector/airMAX _Sector_Antennas_DS.pdf

[11] P. Kabamba and A. Girard, Fundamentals of Aerospace Navigation and Guidance, ser. Cambridge Aerospace Series. Cambridge University Press, 2014. [Online]. Available: https://books.google.com/books?id=5RVQBAAAQBAJ

[12] N. Palumbo, R. Blauwkamp, and J. Lloyd, "Modern homing missile guidance theory and techniques," Johns Hopkins APL Technical Digest, Tech. Rep. 29(1), 2010.

[13] L. Mech and S. Barber, "A critique of wildlife radiotracking and its use in national parks," Biological Resources Management Division, U.S. National Park Service, Fort Collins, CO, Tech. Rep., 2002.

[14] L. Mech, Handbook of Animal Radio-Tracking. Minneapolis, MN: University of Minnesota Press, 1983.

[15] R. Rountree, F. Juanes, and J. E. Blue, "Potential for the use of remotely operated vehicles (rovs) as a platform for passive acoustics," in Listening to Fish: Proceedings of the International Workshop on the Applications of Passive Acoustics in Fisheries, Cambridge, MA, 2002, pp. 138-146.

[16] B. Nielsen and B. Mohl, "Hull-mounted hydrophones for passive acoustic detection and tracking of sperm whales (physeter macrocephalus)," Applied Acoustics, vol. 67, no. 11-12, pp. 1175-1186, 2006.
[17] L. Stutters, H. Liu, C. Tiltman, and D. Brown, "Navigation technologies for autonomous underwater vehicles," IEEE Transactions on Systems, Man, and Cybernetics, Part C: Applications and Reviews, vol. 38, no. 4, pp. 581-589, July 2008.

[18] S. Cowen, S. Briest, and J. Dombrowski, "Underwater docking of autonomous undersea vehicles using optical terminal guidance," in OCEANS '97. MTS/IEEE Conference Proceedings, vol. 2, Oct 1997, pp. 1143-1147 vol.2.

[19] M. Feezor, F. Sorrell, P. Blankinship, and J. Bellingham, "Autonomous underwater vehicle homing/docking via electromagnetic guidance," IEEE Journal of Oceanic Engineering, vol. 26, no. 4, pp. 515-521, Oct 2001.

[20] J. Vaganay, P. Baccou, and B. Jouvencel, "Homing by acoustic ranging to a single beacon," in OCEANS 2000 MTS/IEEE Conference and Exhibition, vol. 2, Providence, RI, 2000, pp. 1457-1462.

[21] D. Wojcik, "Passive localization of underwater acoustic beacons," Master's thesis, Massachusetts Institute of Technology / Woods Hole Oceanographic Institution, Cambridge, MA, 1993.

[22] 3DR Radio V2 Quick Start Guide, 3D Robotics, Inc., Berkeley, California, October 28 2013. [Online]. Available: www.3drobotics.com

[23] Nano Series Operating Manual, v2.9 ed., Microhard Systems, Inc., Calgary, Alberta, Canada, February 2012. [Online]. Available: www.microhardcorp.com

[24] Parrot Bebop Drone, Parrot, SA., 174 quai de Jemmapes 75010 Paris. [Online]. Available: www.parrot.com

[25] iPhone User Guide, Apple, Inc., Cupertino, California. [Online]. Available: www.apple.com

[26] Gerdsen, "Calculating signal strength on telit gprs module," http://www.zombiehugs.com/2011/09/calculatingsignal-strength-on-telit-gprs-module/, Sep. 2011.

[27] J. Tribbeck, "Converting rssi values to dbm," http://support.argogroup.com/article.php?art=387, Sep. 2007.

[28] E. B. Wilson, "Probable inference, the law of succession, and statistical inference," Journal of the American Statistical Association, vol. 22, no. 158, pp. 209-212, 1927. [Online]. Available: http://dx.doi.org/10.1080/01621459.1927.10502953 